combining multiple regional techniques is a safe alternative, using low dose local anaesthetics to reduce chances of LAST. Follow up, monitoring and a multimodal analgesia plan is imperative. More evidence is needed on the effect of inflammation, wound healing, length of stay and chronic pain.

\section{SUCCESSFUL SELECTIVE SENSORY NERVE BLOCKS FOR AWAKE HAND SURGERY USING NERVE STIMULATOR}

A Oiwa*. Toho University School of Medicine, Oomori-Nishi, Ota-ku, Tokyo, Japan

\subsection{6/rapm-2021-ESRA.32}

Background and Aims The selective sensory nerve blocks in awake tendon reconstruction have been started since 2020 in our institute. We managed these surgeries under general anesthesia combined with blocking only sensory nerves under echo guidance.

Methods In this presentation, we would like to present 11 cases and extract our problems. The surgeries were finger tendon repairs and finger/hand joint functional reconstructions. The tourniquet was used during surgery in all cases. General anesthesia was performed by the 'asleep-awake' technique, and the patient was awakened when the tourniquet was released after the tendon repairment.

Results The sites of regional anesthesia were the lateral forearm cutaneous nerve, the medial upperarm/forearm cutaneous nerve, the posterior cutaneous nerve, the distal radial nerve, the forearm interosseous nerve, and the distal ulnar nerve.

$2.5-4 \mathrm{ml}$ of 0.1 to $0.125 \%$ levobupivacaine was used for cutaneous nerves and $1-3 \mathrm{ml}$ of $0.5 \%$ levobupivacaine was used for other nerves. In all 8 cases, it was possible to move their digits during surgery. The most important is, even though we tried to reduce the injection amount and tried more regionally, but in the first 2 cases, the maintenance of finger muscles strength was slightly insufficient. After using the nerve stimulator, this problem was resolved.

Conclusions There was one patient who complained of pain, but it was possible to deal with adding local anesthesia. No vomiting, toxicity or respiratory problems were observed, and no cases abandoned awakening. Hand surgery with selective sensory nerve blocks was a good method with high treatment accuracy.

\section{TEAMWORK AND COMMUNICATION IN THE OPERATING ROOM (OR) - A LOCAL ANESTHESIA (LA) SYSTEMIC TOXICITY CASE REPORT}

1J Castro Nunes, ${ }^{2} \mathrm{G}$ Cruz*, ${ }^{2}$ A Claudia. ${ }^{1}$ Instituto Português de Oncologia, Lisboa, Portugal; ${ }^{2}$ Centro Hospitalar Universitário Lisboa Norte, Lisboa, Portugal

\subsection{6/rapm-2021-ESRA.33}

Background and Aims Local anesthetic systemic toxicity (LAST) is a rare but potentially life-threatening adverse event that occurs after local anesthetic administration through different routes ${ }^{1}$. This case intends to highlight the importance of bidirectional communication in the operating room (OR) and identification of warning signs and symptoms of LAST.

Methods A healthy 22-year-old female (weight $56 \mathrm{Kg}$, height $159 \mathrm{~cm}$ ), with a type III odontoid fracture due to a vehicle roll over was brought to the OR for a halo-vest placement. The procedure was executed under monitored anesthesia care, with the use of local anesthetic (LA) alone, as requested by the surgical team, allowing neurological examination throughout its execution. 2\% lidocaine without adrenaline was administered subcutaneously in the frontotemporal region for pin insertion.

Results After 40 minutes, the patient became agitated, complained of blurred vision, metallic taste, and developed supraventricular tachycardia. When questioned, the surgical team revealed that $30 \mathrm{~mL}$ of lidocaine had been administered. The anesthesia team presumed the complaints were due to LAST and Institutional protocol was implemented. It includes antiepileptic therapy, hemodynamic and ventilatory support and lipid emulsion therapy. Upon termination, the patient was transferred to a post-anesthetic-care-unit and was discharged 24h later without any further complications.

Conclusions Systemic toxicity can be life threatening and rapid identification is key to prevent mortality. Although subcutaneous administration is less prone to toxicity, multiple injections in the scalp, witch is a highly vascularized area, cause a rapid absorption $^{2}$. Bidirectional team communication in the OR is essential for complication prevention, intraoperative differential diagnosis and systematic approach in such critical events.

\section{SUBARACHNOID HEMATOMA AFTER ATTEMPTED SPINAL BLOCK}

RF Santos, RL Silva*, A Gomes, N Ribeiro, F Moura. Centro Hospitalar Tâmega e Sousa, Guilhufe, Penafiel, Portugal

\subsection{6/rapm-2021-ESRA.34}

Background and Aims Subarachnoid hematoma is a rare but potentially serious complication of a subarachnoid block. Its occurrence is associated with several risk factors such as multiple spinal attempts, traumatic puncture and concurrent therapy with anticoagulants and/or antiplatelet agents. ${ }^{1}$

Methods Description of a case report in the section below.

Results Case Report: A healthy pregnant woman was admitted for an elective c-section under subarachnoid spinal block. After two hematic punctures at different lumbar levels, it was decided to proceed with a general anaesthesia instead. Surgery was performed with no reports of complications. About 7 days later, the patient reported neurologic symptoms and a spinal hematoma was diagnosed (figure 1 and 2). After

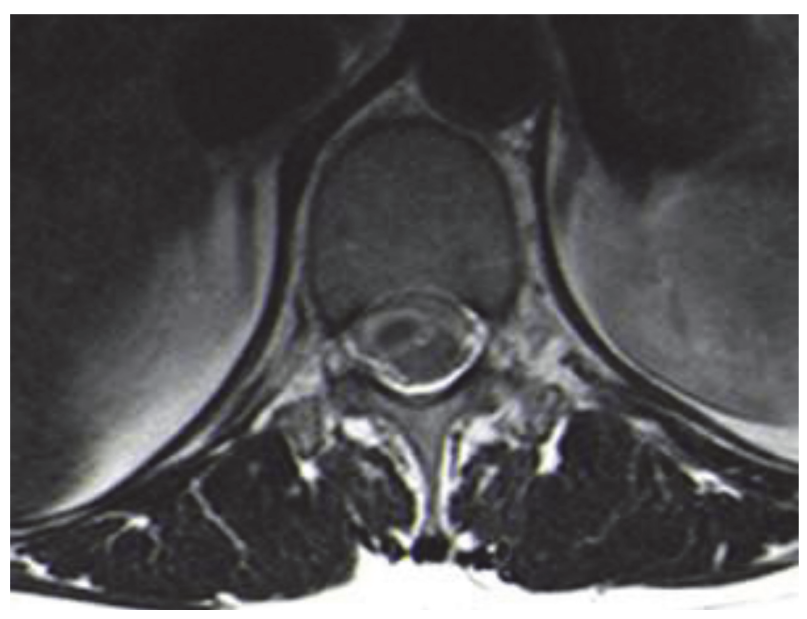

Abstract 34 Figure 1 Dorsolumbar MRI, T2 axial section image of dorsolumbar subarachnoid hematoma with a maximum width of $9 \mathrm{~mm}$ 